\title{
A critical look at the role of AGB stars in stellar population synthesis
}

\author{
A. Lançon \\ Observatoire astronomique, Université L. Pasteur \& CNRS, UMR 7550, \\ 11 rue de l'Université, 67000 Strasbourg, France
}

\begin{abstract}
Asymptotic giant branch stars are essential contributors to the near and mid-IR emission of intermediate age $\left(10^{8}-10^{9} \mathrm{yr}\right.$ old $)$ stellar populations. Detecting this light will set constraints on the star formation history in galaxies and, conversely, the search for AGB signatures in well studied populations will help us reduce some of the still large uncertainties in AGB models. This paper reviews how AGB stars are currently included in population synthesis models and which spectral features can be used to identify their emission in galaxy light; targets for observational tests are suggested, and some observational and theoretical difficulties are discussed.
\end{abstract}

\section{Introduction}

Asymptotic Giant Branch stars (AGB stars) are rare members of stellar populations. However, they are among the most luminous cool stars and can therefore be detected sometimes even individually in external galaxies; they are also the coolest of the luminous stars and hence produce spectral signatures that we may search for in integrated near/mid-IR spectra. Finally, the carbon we are made of and the pre-solar meteorites found on Earth remind us of the role played by AGB stars in the evolution of galaxy matter.

Population synthesis techniques predict the evolution of stellar distributions in the HR diagram and the corresponding integrated emission properties. Nowadays they also keep track of the chemical evolution.

In this review, we will focus on the spectrophotometric aspect of population synthesis, at solar and LMC metallicities. We will discuss how we can predict the contribution of AGB stars to the integrated spectrum and how we can recognise these contributions in observed spectra. Much of this work has become feasible only recently thanks to advances in AGB theory and to more complete samples of AGB observations. We will then suggest extragalactic targets and discuss what the detection - or not - of AGB signatures can tell us about the galaxies or alternatively about AGB evolution. The relevant observations are becoming feasible now and are mostly work of the future. 


\section{The contribution of AGB stars to the bolometric luminosity}

Predicting the contribution $f_{\text {bol }}$ of AGB stars to the bolometric luminosity as a function of time for an evolving stellar population (SP) "only" requires a set of stellar evolution tracks. Such tracks used to be constructed semi-empirically (e.g. Charlot \& Bruzual 1991) and are now provided by so-called synthetic AGB evolution models (Groenewegen \& de Jong 1993; Marigo, this volume). The effects of thermal pulses are taken into account in these calculations, but the product used as input in population synthesis calculations is a set of smooth tracks that needs to be well understood as a representation of either the interpulse properties or some mean properties, e.g. the energy weighted averages over helium shell flash cycles. The precise definition determines further steps in the synthesis code. In the first case for instance, corrections should be applied to account for the post-flash luminosity dip. ${ }^{1}$

The critical parameter in setting $f_{\text {bol }}$ is the mass loss $\dot{M}$, which determines AGB lifetimes and the range of initial masses for which the AGB phase occurs. Other parameters are implicit in $\dot{M}$ : e.g., metallicity influences grain formation and the efficiency of radiation pressure, the mixing parameters and gas opacities influence the extension of the stellar envelopes and the Mira-type pulsation properties, that contribute to starting the winds. Available tracks usually differ in more than one of these parameters, which makes it difficult to interpret differences in lifetimes and energy distributions; at this stage, the scatter among the lifetimes found in the literature for the thermally pulsing AGB (TP-AGB) is about a factor of 2 and must be treated as an uncertainty.

The predictions reported in this paper are based on the population synthesis code of Fioc \& Rocca-Volmerange (hereafter FRV; $Z=0.02$ : 1997, other $\mathrm{Z}:$ 1998). A Salpeter IMF with a lower mass limit at $0.1 \mathrm{M}_{\odot}$ is adopted in all calculations. The TP-AGB evolution is included in the code following the prescriptions of Groenewegen et al. $(1993,1995)$; the resulting tracks follow those of Vassiliadis \& Wood (1993) closely in the HR diagram. The FRV lifetimes are always close to the shortest ones found in the literature, therefore we do not expect to overestimate the effect of the AGB. A more complete study will be reported elsewhere.

After an instantaneous burst of star formation (IB), we find that the contribution $f_{\text {bol }}$ due solely to the TP-AGB stars reaches maximal values $(\gtrsim 10 \%)$ between 0.3 and $\sim 1.5 \mathrm{Gyr}$, corresponding to AGB progenitor masses between 1.7 and $3.5 \mathrm{M}_{\odot}$. At $1.5 \mathrm{Gyr}$ the complete AGB (E-AGB+TP-AGB) contributes about a quarter of the light; values of up to $40 \%$ have been reported (Charlot \& Bruzual 1993). At later stages, the light of the TP-AGB stars is progressively lost in the increasing contribution of E-AGB and first giant branch stars.

AGB lifetimes can in principle be tested with integrated V-K colours since the AGB contribution is essentially emitted at near-IR wavelengths. The TPAGB by itself is responsible for more than $40 \%$ (and up to $60 \%$ ) of the $\mathrm{K}$ band

\footnotetext{
${ }^{1}$ Synthetic evolution was first introduced as a means of simplifying population synthesis calculations; we note however that synthetic models become more and more complex, and that a return to the direct use of the output of full evolutionary calculations may become attractive again.
} 
light 0.1 to 1 Gyr after an IB. At these ages, the red supergiants responsible for very red colours earlier on have died. We refer to Charlot (1996) for an interesting model allowing comparison of colour and AGB contribution predictions.

Girardi \& Bertelli (1998) show how changes in $\dot{M}$ affect V-K; in particular, the increased $\dot{M}$ and hence shorter lifetimes resulting from envelope burning in massive AGB stars may reduce $\mathrm{V}-\mathrm{K}$ by up to 1 magnitude at the earliest AGB-dominated times. Some ambiguities and practical difficulties faced when performing tests based on colours are discussed in Sect.3.2. and 4.

\section{The effect of AGB stars on the near and mid-IR spectrum}

\subsection{Constructing a suitable stellar library}

In order to predict the distribution of AGB light along the IR spectrum and to determine how AGB stars can be recognised among other cool contributors (red supergiants and first giant branch stars), stellar spectroscopic libraries are needed. Commonly used near-IR libraries include the empirical ones of Kleinmann \& Hall (1986), Terndrup et al. (1991), Lançon \& Rocca-Volmerange (1992), the theoretical one of Bessell et al. (1991) and the semi-empirical compilation of Lejeune et al. (1998). None of these considers the pulsating long period variables (LPVs) explicitely, despite the fact that pulsation is known to modify the spectral type-temperature-colour relations and to increase molecular absorption features (Johnson \& Mendez 1970; Bessell et al. 1989), and that basically all the coolest AGB stars are LPVs. As a consequence, near-IR stellar energy distributions (SEDs) of galaxies have been interpreted in terms of a supergiant and giant dichotomy (mainly on the basis of $\mathrm{CO}$ absorption at $2.3 \mu \mathrm{m}$ and of the Ca triplet at $850 \mathrm{~nm}$ ), but no segregation among cool giant populations has been possible.

Lançon \& Wood (1997, hereafter LW97) have obtained 0.5-2.5 $\mu \mathrm{m}$ spectra for $\sim 100$ cool stars, including instantaneous spectra of a sample of O-rich and Crich LPVs observed at various phases. The observations confirm that only LPVs are able to produce the deepest $\mathrm{H}_{2} \mathrm{O}$ bands around 1.4 and $1.9 \mu \mathrm{m}$ (Mouhcine \& Lançon 1998). Other characteristic near-IR molecular bands are those of VO $(1.05 \mu \mathrm{m})$ and $\mathrm{TiO}(1.25 \mu \mathrm{m}$, in the latest spectral types), or of $\mathrm{CN}(1.1,1.4 \mu \mathrm{m})$ and $\mathrm{C}_{2}(1.77 \mu \mathrm{m})$ in C-stars.

Temperatures were assigned to individual Mira-type spectra of LW97 in two ways. On one hand, R. Alvarez compared the energy distribution (nearIR $\mathrm{H}_{2} \mathrm{O}$ bands excluded) with the static giant models of Plez (model version of Alvarez \& Plez 1998). On the other, colour-temperature relations based on angular diameter measurements were used (Feast et al. 1996). Below $3000 \mathrm{~K}$, the first temperatures are systematically higher than the second, the difference increasing up to $\sim 400 \mathrm{~K}$ around $2000 \mathrm{~K}$. The LPV atmosphere models of Bessell et al. 1996 also indicate low temperatures (in the rather rare cases where the fit is satisfactory). The two latter methods are not independent: angular diameter interpretations require model atmospheres. In view of the difficult reconciliation between large observed radii and fundamental mode pulsation (Wood et al., this volume), there may be systematic errors related to the use of current AGB 


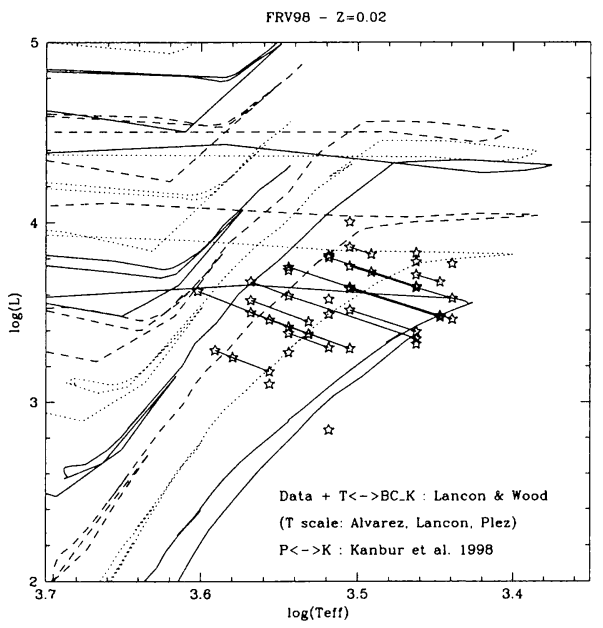

Figure 1. Plotted over the evolution tracks of FRV (1998) are the locations of observed spectra from LW97. $T_{\text {eff }}$ is estimated according to the warmer scale described in the text; luminosities are derived from a $\mathrm{P}-\mathrm{L}_{K}$ relation and the $\mathrm{K}$ band bolometric corrections are measured on the instantaneous spectra themselves ( $\mathrm{K}$ band variability is neglected). Lines connect observations of single stars at various phases.

atmosphere models and the second temperature estimates may be too cool. Therefore, the results presented here are based on an intermediate scale.

The variations of individual stars are illustrated in Fig. 1. Averaging the spectra star by star is not a practical way of obtaining a library for population synthesis: it requires complete phase coverage for each star as well as an estimate of its fundamental parameters (initial mass, metallicity, evolutionary status). The latter are so uncertain that the resulting mean spectra are difficult to order. First attempts with the LW97 library have shown that the expected global spectral evolution from higher to lower temperature is lost in object to object variations. It is more convenient to group the spectra in instantaneous temperature bins. Although the dispersion in the spectral properties inside each bin remains to be explained (Mouhcine \& Lançon, in preparation), this method provides a smoothly evolving sequence.

For population synthesis purposes, the LW97 spectra were combined with the library of Lejeune et al. (1998), as implemented by FRV (1998).

\subsection{Results}

As mentioned above, the AGB spectra that we are able to distinguish from those of older giants are those of pulsating stars.

Fig. 2 shows the predicted behaviour of colours and molecular indices at solar metallicity, with the assumption that the TP-AGB and LPV stages coincide and that LPVs remain visible and O-rich along most of the TP-AGB. The 

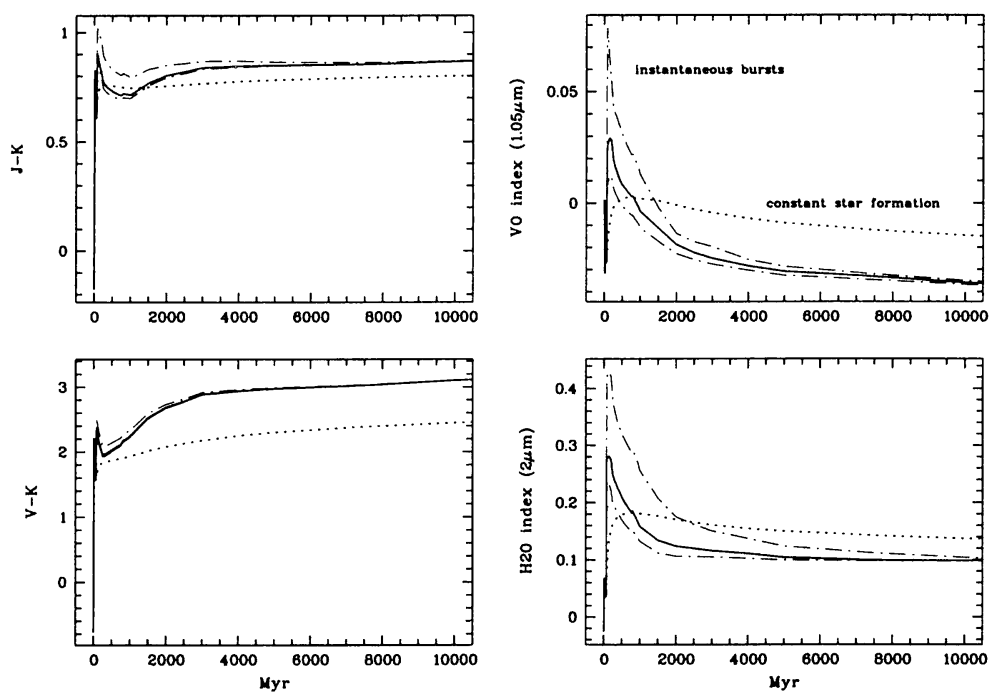

Figure 2. Evolution of selected properties with time. The dot-dashed lines illustrate the burst evolution for the two extreme spectral temperature scales discussed in the text. Molecular indices are in magnitudes.

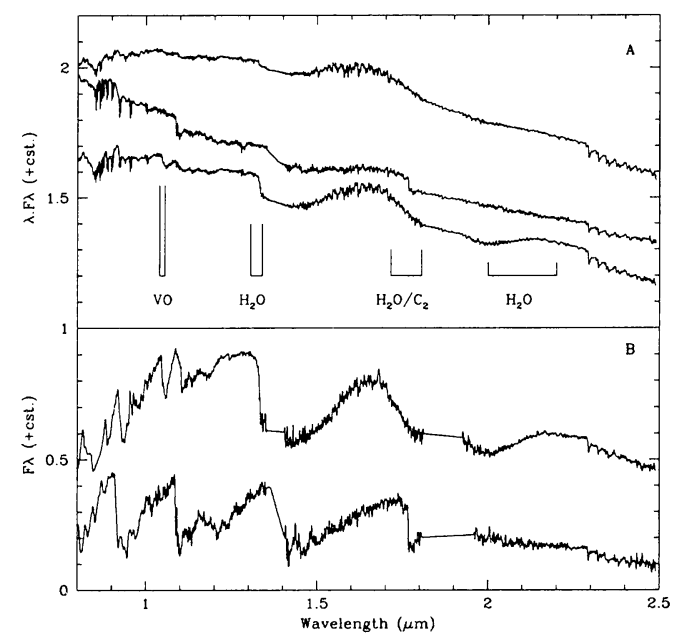

Figure 3. A: selected synthetic integrated spectra. From top to bottom: a static giant dominated $4 \mathrm{Gyr}$ old population, and two $200 \mathrm{Myr}$ old postbursts, when most TP-AGB stars are respectively C-rich and O-rich. Filter centers for useful molecular indices are indicated. B: typical cool O-rich and C-rich LPV spectra. 
blue post-starburst colours are due to the inevitable contamination by luminous intermediate mass blue main sequence stars, coeval with the AGB population.

Several parameters are degenerate in studies based on colours alone: extinction, metallicity and age all redden the integrated spectra, continuous star formation keeps them blue over long times. As an example, the ratio of an IB spectrum at $500 \mathrm{Myr}$ to the spectrum of a constantly star forming population at $2 \mathrm{Gyr}$ is essentially flat between 0.5 and $2.5 \mu \mathrm{m}$. Spectral features must be observed to distinguish the two. As we know from previous models and from observations of star clusters, the Balmer and Paschen series of numerous A type stars characterise the younger of the two populations below $1 \mu \mathrm{m}$. When LPV spectra are included in the models, $\mathrm{H}_{2} \mathrm{O}$ bands appear in the near-IR part of the ratio as broad absorption features at the same relative level as the $\mathrm{H} \alpha$ line (see also Fig. $3 \mathrm{~A}$ ).

The right hand side of Fig. 2 demonstrates that the molecular bands of $\mathrm{H}_{2} \mathrm{O}$ and of VO $(1.05 \mu \mathrm{m})$ unambiguously characterise "postburst populations" 0.1 to $1 \mathrm{Gyr}$ after an IB, with a peak at $150 \mathrm{Myr}$. The maximal numerical values of the molecular indices however depend strongly on the adopted $\mathrm{T}_{\text {eff }}$ scale. In the case of a constant star formation rate, only the warmer $\mathrm{T}_{\text {eff }}$ scale (which leads to the use of spectra with deeper features at a given point of an isochrone) leads to a significant increase of the molecular bands, when compared to the predictions of FRV (1998) without the specific LPV library.

These results remain qualitatively valid in LMC metallicity models.

\subsection{The effects of various model assumptions}

3.3.1 - Does the TP-AGB coincide with the instability strip for LPV pulsation? Period-luminosity (P-L) diagrams of observed Miras suggest that the instability strip is narrower. If we transport the blue edge of the strip into the population synthesis code using the prescription of Vassiliadis \& Wood (1993), we find that the effect on the colours is negligible (LPV spectra are replaced by static giant spectra with very similar energy distributions) but that the depth of the $\mathrm{H}_{2} \mathrm{O}$ features is reduced during the $2 \mathrm{Gyr}$ following an IB (by $\sim 0.03 \mathrm{mag}$ for the index in Fig. 2). VO is not affected: it is produced by the cooler Miras.

In fact, recent data supports a more complex picture (Alvarez et al. 1997; Barthès 1998; Wood et al., this volume; Kiss \& Szatmáry, this volume), that also shows through in theory (Fox \& Wood 1982; Xiong et al. 1998; Tuchman, this volume). Pulsation may be intermittent, depending on phase in the thermal pulse cycle; but globally, AGB stars start varying as small amplitude high overtone pulsators and successively (with overlaps in time) become unstable to lower mode, higher amplitude and longer period pulsation. The Miras of the empirical $\mathrm{P}-\mathrm{L}$ relations are only the largest optical amplitude pulsators. Since we have not excluded low amplitude LPVs from our spectral temperature bins, these bins represent a mixture of pulsation states that may reasonably approximate reality in models based on coinciding TP-AGB and LPV stages.

At the red edge of the LPV instability strip, stars become invisible even in the near-IR due to ejected material, and instead emit longwards of $3 \mu \mathrm{m}$. Bressan et al. (1998) studied the consequences on integrated broad band colours: during the AGB-dominated post-starburst stages, the optical/near-IR spectrum becomes bluer due to the missing late AGB contributions, while the $\mathrm{K}-\lambda$ colours 
with $\lambda>5 \mu \mathrm{m}$ increase dramatically due to $\mathrm{OH} / \mathrm{IR}$ type infrared sources. At LMC metallicities, where the red edge is best defined, we find that V-K changes by a few percent and the molecular features are hardly affected at all. The effect could be stronger at higher metallicities.

3.3.2 - Some AGB stars will end their lives as $\mathrm{C}$ stars, with completely modified spectra. An exploratory study based on the relative C-rich TP-AGB lifetimes of Groenewegen \& de Jong (1993), at the metallicity of the LMC, shows that this totally removes any oxide features apart from $\mathrm{CO}$ for most of the duration of the AGB-dominated post-starburst. Fortunately, the $\mathrm{C}_{2}$ bandhead at $1.77 \mu \mathrm{m}$ then replaces the $\mathrm{H}_{2} \mathrm{O}$ features, and a narrow band photometric index can be defined that measures the time dependent AGB contribution, whether the AGB stars be O-rich or C-rich (Fig. 3; Lançon et al. 1998).

\section{Observational perspectives: where, how and why?}

\subsection{Observational strategies}

The presence of pulsating AGB stars modifies the near-IR spectral signatures and the mid-IR colours during several Gyr after an episode of enhanced star formation and more permanently if star formation continues. However, at the time of this conference the uncertainties on fundamental stellar inputs such as the Mira temperature scale (necessary to connect suitably averaged stellar spectra to evolutionary tracks), the mass-dependent TP-AGB and the LPV lifetimes, the O-star to C-star transition, limit the number of robust predictions directly applicable to extragalactic studies to a few: we expect unambiguous AGB signatures 0.1 to 1 Gyr after a starburst, and we know we may search them using combined near and mid-IR colours (Bressan et al. 1998) or near-IR molecular features (Lançon et al. 1998). Other diagnostic features will certainly appear as soon as the astronomical community will have digested the ISO data. It is important that the most robust predictions be tested observationally before more difficult data interpretations (mixed populations with a complex star formation history) be undertaken.

Significant populations with appropriate ages exist in star clusters of Local Group galaxies, in the bodies of some of these galaxies, in E+A galaxies and in post-starburst galaxies.

Star clusters, in particular the LMC age sequence, may look like ideal targets but each of them contains only a handful of luminous AGB stars. The integrated colours are significantly affected by the stochastic fluctuations in the AGB numbers (Ferraro et al. 1995; Santos \& Frogel 1997; Marigo et al. 1996). Assuming Poisson statistics for these small numbers, we can write the relative, time and wavelength dependent luminosity fluctuations due to TP-AGB stars analytically as:

$$
\frac{\sigma_{L}}{L}=\frac{1}{\sqrt{N}} \cdot \frac{\sqrt{\alpha}\left(L_{a}-L_{r}\right)}{\alpha L_{a}+(1-\alpha) L_{r}}
$$

Here, $N$ is the total number of stars in the population, $\alpha$ is the (small) proportion of TP-AGB stars, $L_{a}(\lambda, t)$ and $L_{r}(\lambda, t)$ are respectively the average emissions per TP-AGB or non-TP-AGB star and are both obtained from population synthesis calculations. With the above mentioned IMF, more than $10^{6}$ stars are required in 
order to keep these fluctuations below $10 \%$ at $\mathrm{K}$ a few $100 \mathrm{Myr}$ after an IB. They will then be 10 times smaller at $0.7 \mu \mathrm{m}$ or in terms of bolometric luminosities, and become negligible at $\mathrm{K}$ within a Gyr.

Nevertheless, now that the AGB numbers in individual Local Group star clusters are known and their effect on the colours has been demonstrated it remains interesting to test whether their near-IR spectroscopic features show through. This requires either several slit positions per cluster, or wide field or imaging spectroscopy.

Larger AGB numbers are present in the bodies of selected nearby dwarf or $\mathrm{E}+\mathrm{A}$ galaxies, but they are superimposed on older populations and their spectral signatures will be severely diluted. If we adopt the maximal value reached by a molecular absorption index in a constant star formation scenario as the threshold for the unambiguous detection of AGB contributions (Fig. 2), we find that postburst populations can be identified as long as they represent more than $\sim 10 \%$ of the total stellar mass in the observed area (Lançon et al. 1998). This result is relatively independent of the SF history of the supposedly old underlying population.

Counts should be used preferentially in the studies of Local Group galaxies (e.g. Gallart 1998), especially since integrated near-IR spectra are still difficult to obtain for extended objects that do not have the high surface brightnesses of currently star forming regions. One of the main observational difficulties is crowding (Martinez-Delgado \& Aparicio 1997). It may also be possible to study AGB contributions with extended "pixel fluctuation methods" (as used in microlens searches, e.g. AGAPE, Ansari et al. 1997) ${ }^{2}$, when pixels integrate the light of less than $10^{6}$ stars.

It follows from the above arguments that the ideal targets for first tests should be both massive and localised, 0.1-1 Gyr old postburst populations. A few such objects have recently been identified: new clusters with estimated stellar masses of up to $10^{7}-10^{8} \mathrm{M}_{\odot}$ form in interacting galaxies and can apparently survive as bound objects for more than the $0.1 \mathrm{Gyr}$ required for AGB stars to appear. Clusters with appropriate estimated ages are listed for NGC 7252, NGC 1275 and NGC 3921 (Miller et al. 1997 and references therein) ${ }^{3}$. Neither dilution nor statistical fluctuations are expected to represent serious hurdles there. Many of the more extended (but still bound) post-starburst areas predicted by the dynamical arguments of Kroupa (1998) would also satisfy the detection criteria.

Before closing this section, we wish to mention observational difficulties. The IR signatures of interest are located near the strong telluric absorption bands of $\mathrm{H}_{2} \mathrm{O}$ and $\mathrm{CO}_{2}$, calling for particularly frequent observations of a calibration star during ground-based observations. High signal-to-noise ratios per (low resolution) spectral element are required to detect molecular absorption bands. On the other hand, dust emission associated with ongoing star formation may hinder mid-IR searches for $\mathrm{OH} / \mathrm{IR}$ emission in places. Spatial resolution must always be sought to limit dilution.

\footnotetext{
${ }^{2}$ An idea first mentioned to me by A.L. Melchior

${ }^{3} \mathrm{~A}$ proposal has been submitted to perform a near-IR search for the predicted AGB signatures
} 


\subsection{Perspectives}

\section{What can we learn about galaxies?}

Once the first tests on populations with known ages will have confirmed the predictions regarding the IR signatures of AGB-rich populations, these observations will be useful in the study of the star formation history of galaxies, the propagation of star formation, the survival of starburst clusters and the potential precursors of globular clusters or dwarf galaxies. Recent star formation is often associated with extinction, in which case optical (e.g. Balmer line) studies present incomplete pictures. Post-starburst knots may be found that coexist with current starbursts in dusty, infrared lumionous objects like NGC 253, IC 342 .

In more evolved objects, late stellar evolution paradoxally helps us understand the UV emission: UV-excesses, previously interpreted as signatures of very recent star formation, are now explained by relatively old AGB-manqués or Hot Horizontal Branch objects, closely related to the stars discussed in this conference (Bressan et al. 1994; Brown et al. 1995; FRV 1998).

Finally, as suggested by Bressan et al. (1998), AGB stars may be used to break the well-known age-metallicity degeneracy of galaxy colours. In brief, the red giant population of galaxies increases with time and with metallicity, leading to similarly red integrated colours in both cases; but while the mid-IR emission of $\mathrm{OH} / \mathrm{IR}$ stars in galaxies is predicted to increase with metal abundance, it decreases with age. A similar argument holds for the near-IR molecular TPAGB signatures, although the predicted effect is small.

\section{What can we learn about the AGB?}

The tests discussed in Sect. 4.1 will provide constraints on AGB lifetimes (i.e. on stellar evolution models), on temperature scales (i.e. on pulsating atmosphere models) and on the O- to C-star transition (i.e. on dredge-up). All these parameters badly need to be better determined.

The unique access to distant populations provided by integrated spectroscopic observations will then allow us to explore AGB evolution in more extreme environments. For instance, it has been claimed that stars with ages of $\sim 0.1 \mathrm{Gyr}$ dominate in the emission of the companion of quasar PG $1700+518$ at redshift 0.29 (Canalizo \& Stockton 1997). Are AGB stars numerous there? Are they carbon-rich or oxygen-rich? While more sensitive infrared instruments are being constructed for high spatial resolution telescopes, we have time to exploit Local Universe data and to improve upon existing AGB model predictions.

Acknowledgments. It is a pleasure to acknowledge the active collaboration of R. Alvarez, M. Fioc, M.A.T. Groenewegen, M. Mouhcine, B. RoccaVolmerange, M. Scholz, D.R. Silva and P.R. Wood in making this review possible.

\section{References}

Alvarez R., Mennessier M.-O., Barthès D., et al., 1997, A\&A 327, 656

Alvarez R., Plez B., 1998, A\&A 330, 1109

Ansari R., Aurière M., Baillon P., et al., 1997, A\&A 324, 843

Barthès D., 1998, A\&A 333, 647 
Bessell M.S., Brett J.M, Scholz M., Wood P.R., 1989, A\&A 213, 209

Bessell M.S., Brett J.M., Scholz M., Wood P.R., 1991, A\&AS 89, 335

Bessell M.S., Scholz M., Wood P.R., 1996, A\&A 307, 481

Bressan A., Chiosi C., Fagotto F., 1994, ApJS 94, 63

Bressan A., Granato G.L., Silva L., 1997, A\&A 332, 135

Brown T., Ferguson H., Davidsen A., 1995, ApJ 454, L15

Bruzual G.A., 1983, ApJ 273, 105

Canalizo G., Stockton A., 1997, ApJ 480, L5

Charlot S., Bruzual G.A., 1991, ApJ 367, 26

Charlot S., 1996, PASP Conf. Ser. 98, 275

Feast M.W., 1996, MNRAS 278, 11

Ferraro F.R., Fusi Pecci F., Testa V., et al., 1995, MNRAS 272, 391

Fioc M., Rocca-Volmerange B., 1997, A\&A 326, 950 (FRV 1997)

Fioc M., Rocca-Volmerange B., 1998, in preparation (FRV 1998; cf. also Fioc M., 1997, PhD thesis, Univ. Paris XI)

Fox M.W., Wood P.R., 1982, ApJ 259, 198

Gallart C., 1998, ApJ 495, L43

Girardi L., Bertelli G., 1998, MNRAS 300, 533

Groenewegen M.A.T., de Jong T., 1993, A\&A 267, 410

Groenewegen M.A.T., van den Hoek L.B., de Jong T., 1995, A\&A 293, 463

Johnson H.J., Méndez M.E., 1970, AJ 75, 785

Kanbur S.M., Hendry M.A., Clarke D., 1998, in A Half Century of Stellar Pulsation: A Tribute to A.N. Cox, eds. P.A. Bradley \& J.A. Guzik, Los Alamos

Kleinmann S.G., Hall D.N.B., 1989, ApJS 62, 501

Kroupa P., 1998, MNRAS 300, 200

Lançon A., Rocca-Volmerange B., 1992, A\&AS 96, 593

Lançon A., Wood P.R., 1997, IAU Symp. 189 on Fundamental Stellar Properties: the Interaction between Observations and Theory, Poster proceedings, ed. T.R. Bedding, Publ.: School of Physics, Univ. of Sydney (http://astro.u-strasbg.fr/ lancon)

Lançon A., Mouhcine M., Fioc M., 1998, in preparation

Lejeune T., Cuisinier F., Buser R., 1998, A\&AS 130, 65

Marigo P., Girardi L., Chiosi C., 1996, A\&A 316, 1

Martinez-Delgado D., Aparicio A., 1997, ApJ 480, 107

Miller B.W., Whitmore B.C., Schweizer F., Fall S.M., 1997, AJ 114, 2381

Mouhcine M., Lançon A., 1998, in Unsolved Problems in Stellar Evolution, Poster proceedings, ed. M. Livio, Space Telescope Sc. Inst.

Santos J.F.C., Jr., Frogel J.A., 1997, ApJ 479, 764

Terndrup D.M., Frogel J.A., Whitford A.E., 1991, ApJ 378, 742

Vassiliadis E., Wood P.R., 1993, ApJ 413, 641

Xiong D.R., Cheng Q.L., Deng L., 1998, ApJ 500, 449 